\title{
ANÁLISE DA ATITUDE DO CONSUMIDOR FRENTE A OBTENÇÃO DE DERMOCOSMÉTICOS
}

\author{
ANALYSIS OF CONSUMER ATTITUDE TOWARDS OBTAINING DERMOCOSMETICS \\ ANÁLISIS DE LA ACTITUD DEL CONSUMIDOR HACIA LA OBTENCIÓN DE \\ DERMOCOSMÉTICOS
}

\author{
Amanda Evelyn Lima da Silva ${ }^{1}$ \\ Rebeca Rocha Coutinho Gomes ${ }^{2}$ \\ José Fernando de Araújo Neto ${ }^{3}$
}

RESUMO: Objetivo: Brasil é o quarto maior mercado mundial de cosméticos, e neste contexto, o mercado de dermocosméticos encontra-se em grande expansão. Método: Este trabalho se trata de uma revisão de literatura com o objetivo geral de avaliar o comportamento do consumidor em relação ao consumo de dermocosméticos. O mercado vem crescendo a cada dia não só pela estética, mas também pela melhora da saúde física e mental dos consumidores. Resultados: Para alcançar o objetivo geral, foram estabelecidos como objetivos específicos definir as principais características de cosméticos e dermocosméticos, identificar e classificar os principais ativos usados na formulação de dermocosméticos e analisar os fatores que influenciam o comportamento dos consumidores frente ao consumo de dermocosméticos. Considerações finais: Concluindo que grande parte da população não conhecem o termo e adquirem os dermocosméticos por conta própria, sendo assim tem a necessidade de haver no estabelecimento um profissional capacitado para orientar o consumidor em relação ao uso de dermocosméticos.

Palavras-chave: Cosméticos. Dermocosméticos e Consumidores.

ABSTRAT: Objective: Brazil is the fourth largest cosmetics market in the world, and in this context, the dermocosmetics market is booming. Method: This work is a literature review with the general objective of evaluating consumer behavior in relation to the consumption of dermocosmetics. The market is growing every day not only for aesthetics, but also for the improvement of physical and mental health of consumers. Results: To achieve the general objective, specific objectives were established to define the main characteristics of cosmetics and dermocosmetics, identify and classify the main actives used in the formulation of dermocosmetics and analyze the factors that influence the behavior of consumers regarding the consumption of dermocosmetics. Final considerations: Concluding that a large part of the population does not know the term and purchase dermocosmetics on their own, so there is a

\footnotetext{
I Graduanda do curso de Farmácia da Faculdade Unifacs. E-mail: amandaevely.lima@gmail.com.

${ }^{2}$ Graduanda do curso de Farmácia da Faculdade Unifacs. E-mail: rebeccarochazooo@outlook.com.

${ }_{3}^{3}$ Doutorado em Química pela Universidade Federal da Bahia. Mestrado em Química pela Universidade Federal da Bahia.Graduado em Farmácia pela Universidade Federal da Bahia. E-mail: jose.araujo@unifacs.br.
} 
need for a qualified professional in the establishment to guide consumers in relation to the use of dermocosmetics.

Keywords: Cosmetics. Dermocosmetics and Consumers.

RESUMEN: Objetivo: Brasil es el cuarto mercado de cosméticos más grande del mundo y, en este contexto, el mercado de los dermocosméticos está en auge. Método: Este trabajo es una revisión de la literatura con el objetivo general de evaluar el comportamiento del consumidor en relación al consumo de dermocosméticos. El mercado crece día a día no solo por la estética, sino también por la mejora de la salud física y mental de los consumidores. Resultados: Para lograr el objetivo general, se establecieron objetivos específicos para definir las principales características de la cosmética y dermocosmética, identificar y clasificar los principales activos utilizados en la formulación de dermocosmética y analizar los factores que influyen en el comportamiento de los consumidores frente al consumo de dermocosmética. Consideraciones finales: concluir que una gran parte de la población desconoce el plazo y compra dermocosmética por su cuenta, por lo que es necesario un profesional cualificado en el establecimiento para orientar a los consumidores en relación al uso de dermocosméticos.

Palabras clave: Cosmética. Dermocosmética y Consumidores.

\section{INTRODUÇÃO}

Os cosméticos eram conhecidos como um produto que tinha um propósito de embelezar, higienizar e proteger com as substâncias ativas que permanecem na camada córnea da epiderme. Os dermocosméticos são cosméticos que tem uma comprovação científica da segurança e eficácia, sendo um aditivo de uso tópico agindo nas camadas mais profundas da pele.

Os produtos são disponíveis para os consumidores, porém na escolha muitos não sabem diferenciar o objetivo para o tratamento. Com a variedade de produtos os clientes estão muito preocupados com o cuidado da saúde e beleza. O comportamento do consumidor envolve vários fatores externos e internos do indivíduo considerando que não é algo estável, pois com a aprendizagem, além de garantir o conhecimento para melhores escolhas são compartilhadas experiências com outros indivíduos.

O trabalho visa contribuir de uma forma geral para que os farmacêuticos conheçam os hábitos de consumo da população mediante a variedade de dermocosméticos que existem no mercado, para que os profissionais possam suprir as necessidades dos consumidores. Com o conhecimento da população sobre os dermocosméticos e o hábito de consumo, os 
farmacêuticos serão capazes de prestar orientações sobre o uso seguro e eficaz dessas formulações, atuando como motivadores do uso dos dermocosméticos.

De maneira geral este trabalho teve como objetivo avaliar o comportamento do consumidor em relação ao consumo de dermocosméticos, entre os objetivos específicos podese citar: definir as principais características de cosméticos e dermocosméticos, fatores que influenciam o comportamento dos consumidores e comportamento dos consumidores frente a obtenção de dermocosméticos.

O tipo de pesquisa a ser realizada neste trabalho será uma Revisão de Literatura narrativa, sendo realizada consulta de livros, dissertações e em artigos científicos através do Google Acadêmico, Scientific Eletronic Library Online (SciELO), PubMed e serão incluídos artigos científicos e demais fontes que abordem o conteúdo proposto, publicados de 2005 até 2019 e que tenham sido escritos nos idiomas português, inglês e espanhol. Foram utilizados como critérios de exclusão do trabalho conteúdos que não estejam relacionados ao tema, fora do período mencionado de publicação ou em outros idiomas que não sejam os listados. As palavras-chave utilizadas na busca são: dermocosméticos (dermocosmetics), cosméticos (cosmetics) e comportamento do consumidor (cosmetics and consumer behavior).

\section{REVISÃO DE LITERATURA}

\section{COSMÉTICOS E DERMOCOSMÉTICOS}

O mercado de cosméticos apresenta grandes avanços tecnológicos de interesse para diversos consumidores de ambos os sexos. Para que exista uma eficácia e segurança do produto deve-se garantir que se siga as normas de regulação dos cosméticos, verificando as etapas, da aquisição de matéria prima até o controle de qualidade final (SANFELICE, TRUITI, 20I0).

De acordo com Galembck e Csordas (2015) a indústria de cosméticos é de suma importância para a economia de vários países, incluindo o Brasil. O crescimento das empresas contribuiu para o aumento de rendas e geração de empregos do país. A inovação de produtos tem a visão de sustentabilidade e utilização de produtos naturais como estratégias de inovação na cosmetologia. O aprimoramento de produtos utilizando óleos vegetais, frutas e sementes como fonte de matéria prima é uma das principais formas de atrair os consumidores. 
O mercado de cosméticos e beleza tem o objetivo de proporcionar prazer e bem-estar, garantindo resultados e qualidade de vida. $\mathrm{O}$ mundo vem passando por diversas mudanças que provocam uma revolução no consumo mundial, sendo voltadas para o reencontro do equilíbrio do corpo e da mente, para a busca do bem-estar e recuperação da qualidade de vida.

Nas últimas décadas a progressão cientifica e tecnológica vem crescendo cada vez mais, por isso fabricantes tem a necessidade da inovação sempre procurando satisfazer as necessidades dos consumidores, tendo gerado uma variedade de produtos. Existe uma variedade de conceitos relacionado ao setor de cosméticos e dermocosméticos, em algumas dessas definições consideram a perfumaria e higiene corporal e em outras consideram independentes (ZUCCO, SOUSA, ROMEIRO, 2012). A pele é um órgão complexo com múltiplas funções de defesa de barreira, de síntese química e de eliminação. Sendo o mais vasto de todos é um invólucro que protege o indivíduo situado entre o meio interior e exterior do nosso corpo. Desde as antigas civilizações eram conhecidos produtos de preparações naturais com finalidade de embelezamento, tornando o principal objetivo do cosmético melhorar a aparência do indivíduo. Desenvolveu-se uma "cosmética preventiva”, que mantém o estado fisiológico da pele ou de restaurar em caso de perturbação. O campo de aplicação da cosmética é alargado e os limites com a dermatologia podem parecer difíceis de serem delimitados.

Os cosméticos são substâncias ou preparados que se destinam nas partes superficiais do corpo, como epiderme, sistemas pilosos e capilares, unhas, lábios e mucosas bocais com o objetivo de perfumar ou proteger mantendo um bom estado de modificação do seu aspecto corporal sem ação ou fins terapêuticos (MINISTÉRIO DA SAÚDE, 1986).

A RDC 2II/os da Anvisa estabelece a definição e a classificação, entre outros produtos, dos cosméticos. Esse grupo é dividido em Grau i e Grau 2 sendo produtos de higiene pessoal, cosméticos e perfumes cuja formulação cumpre com a definição adotada no item I do Anexo I desta Resolução. O grau I é caracterizado por possuírem propriedades básicas ou elementares, não necessita de informações detalhadas quanto ao seu modo de usar e suas restrições de uso, devido às características intrínsecas do produto. O grau 2 possuem indicações especificas, cujas características exigem comprovação de segurança e eficácia, é necessita de informações detalhadas quanto ao modo de usas e suas restrições de uso. 
A ocorrência de efeitos não desejados devido ao uso inadequado, formulação, finalidade do uso, destino da área do corpo, foram estabelecidos critérios para esta classificação. Para comercialização dos produtos de Grau 2 devem ser registrados e aprovados pela Anvisa ( $\mathrm{RDC} \mathrm{N}^{\circ}$ 211/2005).

Quadro I. Classificação de produtos cosméticos

e higiene pessoal.

Produtos Grau tipo I

Aromatizante bucal, Base facial/corporal (sem finalidade fotoprotetora), Baton labial e brilho labial (sem finalidade fotoprotetora), Blush/Rouge (sem finalidade fotoprotetora), Condicionador/Creme rinse/Enxaguatório capilar (exceto com ação antiqueda, anticaspa e/ou outros benefícios específicos que justifiquem comprovação prévia), corretivo facial (sem finalidade fotoprotetora), Creme, Loção e Gel para o rosto (sem ação fotoprotetora e com finalidade exclusiva de hidratação), Creme, Loção, Gel e Óleo para as pernas (com finalidade exclusiva de hidratação e/ou refrescançia), Demaquilantes,

Desodorantes (exceto os com ação
antitraspirantes), Delineador para lábios, olhos
e sobranceiras, Máscara para cílios,
Modelador/fixador para sobranceiras,
Removedor de esmalte, Secante de esmalte,
Sombra para pálpebras, Protetor labial sem
protetor, Produtos para fixar, modelar e/ou
embelezar os cabelos: fixadores, laquês,
reparadores de pontas, óleo capilar, brilhantina,
mousse, creme para modelar e assentar os
cabelos, máscara capilar e umidificar capilar.
Clareador da pele, Clareador para as unhas
químico, Bronzeador simulatório, Água
oxigenada io a 40 volumes (incluindo as
cremosas exceto as de uso medicinal),
Bronzeador, batom labial (com ação
preenchedor), Antitranspirante,
Antitranpirante pédico, Ativador/Acelerador de
bronzeado, Condicionador infantil, Dentifrício
anticaria, antitártaro e antiplaca, Depilatório
químico, desodorante antitranpirante axilar,
Fixador de cabelo infantil, Maquiagem com
fotoprotetor, Produto de limpeza/ higienização
infantil, Produtos para rugas, Protetor solar,
Protetor solar infantil, Removedor de cutículas,
Repelente de insetos, Sabonete antisséptico,
Sabonete antiacne, Creme ( com ação
regeneradora) Tintura capilar
temporária/progressiva/permanente, Shampoo
anticaspa/antiqueda e Shampoo colorante,


Fonte: $\left(\mathrm{RDC} \mathrm{N}^{\circ} 2 \mathrm{II} / 2005\right)$.

Dermocosméticos são definidos como produtos que atuam além da epiderme, possuindo indicações específicas e desenvolvidas a partir de estudos científicos e clínicos (Matos, 2015). São produtos de venda livre, algumas substâncias podem ser absorvidas pela pele e podem alcançar a circulação sistêmica. Sendo assim, o uso deve ser com orientação de um dermatologista ou um farmacêutico, pois são considerados cosméticos de grau de risco 2 pelos órgãos reguladores brasileiros (RESOLUÇÃO DA RDC $\mathrm{N}^{\circ}$ 07, DE Io DE FEVEREIRO DE 2015).

Os dermocosméticos possuem bioativos como os ácidos e vitaminas com ação na região aplicada, já os cosméticos de tratamento são utilizados por profissionais da estética. A indústria da beleza vem investindo em cosméticos que relacionam o estético e saúde. A vitamina $C$ ou ácido ascórbico e o ácido hialurônico são dois ativos manipulados em dermocosméticos que estão presentes em tratamentos estéticos e uso home care, onde ambos são substâncias que atuam na revitalização, diminuição de radicais livres em excesso, hidratação, flacidez e no clareamento de manchas (BERLINCK, 2015).

\section{FATORES QUE INFLUENCIAM O COMPORTAMENTO DOS CONSUMIDORES}

\section{FATORES DE MERCADO}

Em qualquer país, o fator econômico altera o cenário do mercado de maneira direta, onde o estado geral econômico influencia o comportamento do consumidor, afetando o senso de otimismo e a disposição de compra. O cenário de um país em relação ao salário, níveis de emprego e crédito para consumo, um dos principais estímulos do meio que alteram o comportamento do consumidor, gerando aumento ou diminuição do poder de aquisição e 
influenciando na disposição de compra, por meio do sentimento do otimismo levando o consumidor a antecipar ou adiar as suas compras.

O ambiente econômico influencia no comportamento de compra do consumidor, levando aos ciclos econômicos, quando economia vai bem crescem o emprego e a produção, aumentando os gastos dos consumidores, tendo uma demanda maior, aumentando o preço gerando inflação. Tendo a inflação eleva a taxa de juros, tornando o consumidor mais conservador e reduzindo os gastos. Com a diminuição dos gastos, elevam o estoque reduzindo a produção, gerando o desemprego, reduzindo ainda mais o consumo. Tendo a redução dos estoques, os fabricantes retornam ao nível de produção, com a retomada na economia iniciando um novo ciclo.

A política governamental (monetária, fiscal e pública) influencia o consumidor no ato da compra. A fiscal regula as práticas comerciais e aquisição do mercado governamental. A pública ao estabelecer regulamentações sobre o produto, fusão e aquisição pode favorecer ou inibir o consumo, produção de produtos e serviços. A monetária influencia no ajuste na taxa de juros, estimulando o consumo com ações restritivas (PROFESSORES DO DEPARTAMENTO DE MERCADOLOGIA DA FGV-EAESP E CONVIDADOS, 2004).

\section{FATORES CULTURAIS}

A cultura vem influenciando a sociedade desde gerações passadas, sendo um centro complexo de valores e crenças que ao decorrer dos anos vem se tornando passado e reforçando através de instituições como escolas e igrejas. O que consumimos como: alimentos, vestimentas e músicas e até mesmo no que acreditamos depende da diversidade e crença da nossa cultura (PROFESSORES DO DEPARTAMENTO DE MERCADOLOGIA DA FGV-EAESP E CONVIDADOS, 2004).

A influência da cultura sobre o consumismo pela sociedade é reconhecida e grande parte do comportamento do consumidor se integra no fator cultural. Entretanto por mais que se admita que a cultura tem um efeito direto sobre a sociedade, não se sabe identificar a real origem desta influência. Contudo, por mais óbvio que isto seja várias empresas tiveram lançamentos de produtos fracassados pelo fato de não ter tido o cuidado de conhecer um pouco da cultura. Portanto ainda que não venha afirmar que os valores não tenham impacto 
sobre o comportamento de compra e consumo, é destacado que sua influencia sobre a atitude dos consumidores é importante e que os valores de fato tem impacto sobre o processo de escolha e decisão do indivíduo.

A cultura é um dos fatores mais importantes que devem ser levados em consideração no planejamento, desenvolvimento dos produtos e na escolha da definição do marketing, já que isso pode implicar diretamente na vida de um indivíduo em sociedade. Vários fracassos ocorrem no marketing pela falta de sensibilidade, em não levar em consideração a realidade cultural em que se encontram os consumidores (PINHEIRO, CASTRO, SILVA, NUNES,2006).

\section{FATORES SOCIAIS}

O processo que ocorre entre dois ou mais indivíduos, onde a ação de um deles é ao mesmo tempo resposta ao outro indivíduo e estímulo para as ações destes é chamada de interação social. A ação de um são os resultados e a causa das ações do outro, sendo assim as motivações, percepções e atitudes dos consumidores é um ponto de partida lógico, onde as decisões de compra são fatos ligados à indivíduos (KARSAKLIAN, 2008).

O comportamento do ser humano é sólido na aprendizagem, proporcionado pela interação social entre as pessoas, onde este contato se transforma numa relação psicológica tornando-se um grupo de referência (PROFESSORES DO DEPARTAMENTO DE MERCADOLOGIA DA FGV-EAESP E CONVIDADOS, 2004).

Os grupos de referência exercem alguma influência diretamente ou indiretamente sobre as atitudes ou comportamentos de indivíduo. A influência direta, são os grupos de afinidades, que interagem de forma contínua e informalmente, como primário: a família, amigos, vizinhos e colegas de trabalho. E os que interagem de maneira formal e menor interação contínua são os grupos religiosos e profissionais ou associações de classe. Os grupos influenciam de forma profunda na vida das pessoas, expondo novos comportamentos e estilos de vida, influenciando as atitudes e sua autoimagem, afetando nas escolhas reais do produto e marca. As pessoas também são influenciadas por grupos nos quais não fazem parte, como os grupos de aspiração os quais se espera pertencer e grupo de dissociação onde cujos valores ou comportamentos são rejeitados (KOTLER, KELLER, 2006). 
Em cada grupo existe uma função ocupada por cada membro, sendo assim a posição ocupada é definida pelo conjunto de direitos e deveres do indivíduo no grupo, com um valor diferencial de cada posição. A importância atribuída a cada posição é indicada por símbolo de status, tanto nas sociedades mais desenvolvidas como nas primitivas. O grupo influencia na aquisição do produto e escolha de marca, percebe-se que a situação de consumo é por vezes mais importante do que o próprio produto. (MINIARD E COHEN, I983 apud KARSAKLIAN, 2008).

\section{FATORES PESSOAIS}

Os fatores pessoais também influenciam nas decisões dos consumidores, como idade, personalidade, circunstâncias econômicas, estilo de vida, valores, ocupação, autoimagem e estágio no ciclo de vida. Como estes fatores tem impacto direto no comportamento do consumidor, é determinante para os profissionais de marketing conhecê-los bem (KOTLER, KELLER, 2006).

As pessoas tem um grande potencial de consumo de produtos e serviços durante a vida. O padrão de consumo é moldado com o ciclo de vida da família e com o número, sexo e idade de todos os membros em qualquer ponto do tempo.

O ciclo de vida de uma família tem diversas formas, sendo como a família pode ser estruturada ao longo da vida de uma pessoa e o comportamento de compra associado a cada ciclo. Sendo um fator muito importante, pois é um critério de segmentação mais utilizado pelos profissionais de marketing (PROFESSORES DO DEPARTAMENTO DE MERCADOLOGIA DA FGV-EAESP E CONVIDADOS, 2004).

\section{FATORES PSICOLÓGICOS}

$\mathrm{O}$ ato de comprar tem como principal ponto de partida a motivação para atender as necessidades despertando o desejo que é atendido de forma específica tendo uma determinada preferência. A motivação,percepção, aprendizagem e memória influenciam na reação do consumidor a vários estímulos de marketing.

$\mathrm{Na}$ motivação, as forças psicológicas que formam o comportamento do indivíduo são inconscientes e que ninguém chega entender por completo as próprias motivações. Quando a 
pessoa leva em consideração a marca ela reage não apenas a possibilidade declarada dessas marcas, mas também a forma, o tamanho, o peso, o material, a cor e o nome estimulando sensações. Já na percepção, a pessoa motivada está pronta para a agir, sendo influenciada pela percepção da situação, onde a percepção é o processo no qual o indivíduo seleciona, organiza e interpreta algumas informações recebidas, dependendo não apenas dos estímulos físicos mas também da relação entre estímulos com ambiente das condições internas da pessoa.

A aprendizagem influência no comportamento do consumidor, pois quando a pessoa age ela aprende. A mudança de comportamento de uma pessoa está na aprendizagem, sendo decorrente da experiência por parte do comportamento humano. Já na memória desempenha um papel importante, pois todas as informações e experiências ocorridas pelas pessoas ao longo da vida podem acabar em sua memória de longo prazo (KOTLER, KELLER, 2006).

Figura I - Fatores que influenciam o comportamento dos consumidores

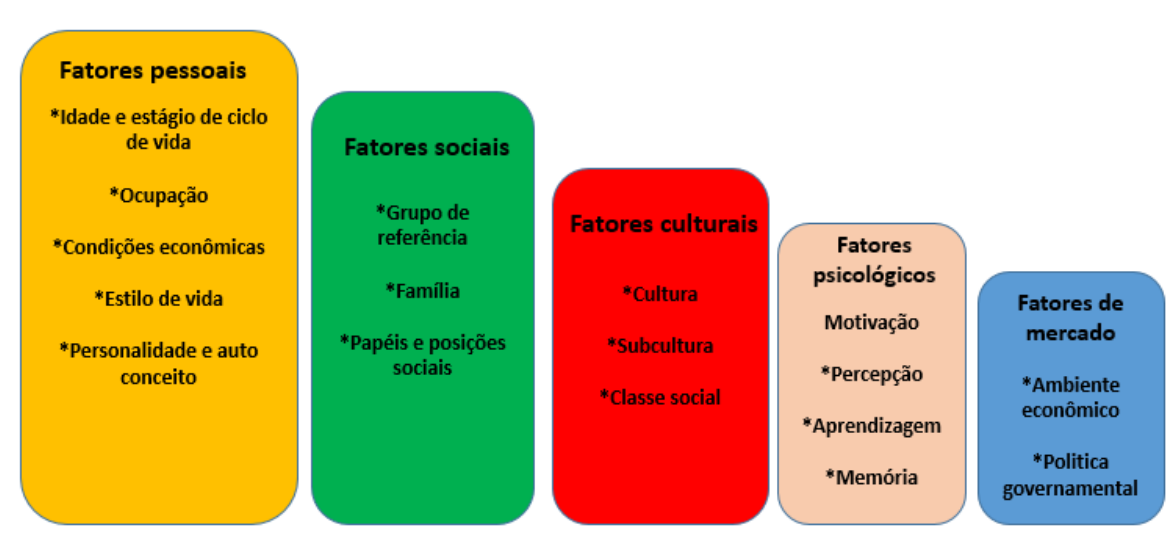

Fonte: (PROFESSORES DO DEPARTAMENTO DE MERCADOLOGIA DA FGV-EAESP E CONVIDADOS, 2004).

\section{COMPORTAMENTO DO CONSUMIDOR NA OBTENÇÃO DE DERMOCOSMÉTICOS}

O comportamento é representado pelas atividades mentais e emocionais realizadas na compra e uso de produtos para a satisfação de necessidades e desejos (RICHERS, 1984). É considerado um campo multidisciplinar, pois tem a ver com o pensamento e pesquisa, tendo ideias de diversas áreas como, aprendizado, motivação, percepção, atitude, personalidade, grupos sociais e classes (LACERDA, 2007). 
Para entender o comportamento do consumidor é necessário compreender o que é e quem são os consumidores. Para Enis (1983), comportamento é a relação feita entre estímulos e respostas. Já para Lima (2009) o comportamento é entendido como as reações de um indivíduo perante a sociedade, com ações, sentimentos e fala.

O comportamento é caracterizado pelo processo a escolha produto até o seu consumo. As empresas tentam encontrar uma relação entre as diferenças de consumo, com base na particularidade das personalidades de seus clientes, para oferecer aquilo que eles desejam. $\mathrm{O}$ estudo do comportamento envolve diversas atividades que transpassam a percepção da necessidade até o momento do descarte do produto

O comportamento do consumidor é formado por diversos processos envolvidos no ato em que a pessoa escolhe, compra, usa e descarta, ideias ou experiências, a fim de satisfazer suas necessidades e desejos. No ato da compra varia quanto ao tipo de produto que o consumidor pretende adquirir. É indispensável que cada mercado entenda quais os desejos e necessidades dos consumidores para obtenção do produto, pois apresentam particularidades diferenciadas (SOLOMON, 20II).

Os consumidores comparecem cada vez mais aos mercados e sofrem influências de informações sobre produtos as quais trazem expectativas e desejos. Atualmente, os cosméticos têm um mercado de destaque mundial. Isso deve-se a participação dos consumidores destes produtos de forma ativa e constante, pois passaram a usá-los com maior frequência em seu dia a dia. Esse tipo de produto tornou-se motivo de pesquisas em diversos campos de estudo, principalmente no marketing.

O Brasil é o terceiro maior consumidor de produtos de higiene e beleza do mundo, o crescimento mostra que a população se preocupa com sua aparência e higiene pessoal cada vez mais. Dessa forma, é necessário que as emprestam tenham conscientização para melhorar a qualidade dos produtos e atrair novos clientes (ABIHPEC, 2009).

Quando uma necessidade é despertada o consumidor tem a vontade de satisfazê-la. Dessa forma, quando as necessidades geram uma tensão, impulsiona o consumidor a tentar reduzi-las ou eliminá-las. A principal influência para a motivação do ser humano é a necessidade, pois persistem até serem saciadas. Entre várias necessidades dos consumidores, 
três principais destacam como, associação (estar acompanhado de outra pessoa), poder (controlar o meio) e singularidade (garantir a identidade individual) (SOLOMON, 20II).

O mercado de cosméticos brasileiro tem se expandido cada vez mais, sendo reconhecido e utilizado por consumidores de todas as classes sociais, pois os produtos deixaram de ser vistos como fúteis e passaram a ser vistos como essenciais. Sendo assim, as pessoas estão se preocupando mais com sua beleza e bem-estar (SÁ, 2010).

$\mathrm{Na}$ sociedade as mulheres são mais propensas a gastar com produtos cosméticos, onde preferem seguir indicações de amigos a indicações médicas, sendo o contrário dos homens. O comportamento dos dois gêneros é muito presente na sociedade atual, sendo possível visualizar um crescente número de revistas e publicações na internet para difundir o uso de cosméticos entre o público masculino (SÁ, 2010).

Sobre a fidelidade dos consumidores, os produtos são adquiridos de marcas variadas, onde os consumidores são poucos fiéis. $\mathrm{O}$ consumo dos produtos ocorre mais nas drogarias, tendo uma necessidade de o farmacêutico estar atualizado sobre o tema de modo a atender a necessidade do consumidor. Os consumidores relatam efeito positivo com o uso do cosmético e dermocosméticos e estão dispostos a pagar mais pelos dermocosméticos que pelos cosméticos (SOLOMON, 20II).

Para o aumento de adesão dos pacientes deve-se compreender quais as marcas preferidas dos consumidores, auxiliando na prescrição de produtos dermocosméticos. Algumas marcas importam-se com estudos clínicos de eficácia, com o conhecimento da forma de produção e a integridade da empresa, aliado às preferências do mercado consumidor, faz com que a adesão ao tratamento seja mais eficaz (SCHMITT, 2002).

A publicidade é um fator principal que influenciam na compra de cosméticos e dermocosméticos aliado à noção de imagem corporal. $\mathrm{O}$ preço tem uma grande influência na aquisição de produtos estando aliada ao fato de que as pessoas levam em consideração indicações de amigos na hora da compra, deixando de ser fiéis as marcas se encontrarem melhores produtos em empresas concorrentes. As marcas criam valores de longo prazo através de seus nomes e associações a um produto, proporcionam uma imagem, asseguram qualidade, oferecem soluções definitivas, ultrapassam os elementos específicos do produto (SCHMITT, 2002). 
Cobra (1992) define marca como "um nome, sinal, símbolo, design, ou combinação de tudo isso". Tem o objetivo de identificar o produto para diferenciar da concorrência. A marca faz parte do composto de produtos e é um fator importante na adaptação para satisfação das necessidades dos consumidores.

Para a escolha de uma marca considera-se alguns critérios como compreensão dos consumidores, facilidade de pronúncia, memorização, associado a imagem do produto, ter eficácia, adaptar-se a embalagem, disponível para uso e possuir pronúncia globalizada em várias línguas (COBRA, 1992).

\section{RESULTADOS E DISCUSSÕES}

Os produtos são utilizados por ambos os sexos, mas muitos não tem o conhecimento dos termos de cosméticos e dermocosméticos. O fator que mais influencia no ato da compra é a qualidade do produto e indicação de conhecidos, o que demonstra um mercado consumidor cada vez exigente quanto aos produtos.

Referente a motivação da compra de produtos cosméticos e dermocosméticos, os consumidores utilizam os cosméticos para o bem estar, melhorar na aparência e prevenção. Já no caso dos dermocosméticos é utilizado para tratamento, melhora na aparência e prevenção de alterações na pele, unha e cabelos.

Muitos consumidores na hora da compra para aquisição dos produtos, são adquiridos por conta própria, prescrição de dermatologista e do farmacêutico. Em relação aos dermocosméticos, ocorreram por prescrição do dermatologista, dos produtos adquiridos por conta própria e por prescrição do farmacêutico. Para comprar um cosmético ou dermocosméticos não é necessário ter receita médica, mas a maioria das vendas dos dermocosméticos são por recomendação médica ou de um dermatologista. Ambos os produtos são de venda livre (LACERDA, 2007).

Os consumidores relatam efeito positivo com o uso de cosméticos e dermocosméticos e que estão dispostos a pagar mais pelos dermocosméticos que pelos cosméticos, o que evidencia a ascensão do mercado de dermocosméticos, tendo um número de vendas elevados. Para melhores resultados, é importante que as drogarias realizem ações e campanhas nos locais de 
compra, além de estar cada vez mais próximas dos médicos e dermatologistas para que esses divulguem e indiquem seus produtos aos pacientes.

\section{CONSIDERAÇÕES FINAIS}

O presente trabalho teve o objetivo de avaliar o comportamento do consumidor na obtenção de dermocosméticos. O avanço do mercado acarreta no uso de diferentes cosméticos e despertam novos anseios e necessidades dos consumidores. $O$ estudo proporcionou uma maior compreensão acerca do conhecimento sobre cosméticos e dermocosméticos, onde ambos são de venda livre, tornando-se necessário que os profissionais Farmacêuticos tenham um maior conhecimento sobre os produtos comercializados a fim de atender os clientes de forma mais eficiente.

Sendo possível identificar os principais fatores que mais influenciam na compra dos produtos, levando em consideração os processos de aprendizagem para a aquisição, são os pessoais, sociais, culturais, psicológicos e de mercado. Conclui-se que fatores motivacionais externos e o processo de aprendizagem prevalecem na escolha e utilização de cosméticos.

Os resultados e conhecimentos gerados tem contribuição não apenas para o campo científico, em virtude das referências bibliográficas que servem de fonte de informação na implementação de pesquisas adicionais, mas também à comunidade empresarial, por causa do caráter prático. As discussões são úteis na busca de um melhor entendimento do comportamento do consumidor e suas preferências quanto aos produtos.

\section{REFERÊNCIAS}

ABIHPEC. Faturamento de Higiene Pessoal, Perfumaria e Cosméticos cresce 12,6\% em 2010.

AGÊNCIA NACIONAL DE VIGILÂNCIA SANITÁRIA (ANVISA). RDC o7. Dispõe sobre os requisitos técnicos para a regularização de produtos de higiene pessoal, cosméticos e perfumes e dá outras providências. Brasília, 2015. 
AGÊNCIA NACIONAL DE VIGILÂNCIA SANITÁRIA (ANVISA). RDC 2Ir. Ficam estabelecidas a Definição e a Classificação de Produtos de Higiene Pessoal, Cosméticos e Perfumes, conforme Anexos I e II desta Resolução. Brasília, 2005.

BERLINCK, Nathália Sorroche. Formulação de produtos dermocosméticos com aplicação em procedimentos estéticos. 2015. $88 \mathrm{f}$, , 2015.

COBRA, Marcos. Administração de Marketing: A análise do mercado de consumo e o comportamento do consumidor. $2^{-}$ed. São Paulo: Atlas, 1992;

ENIS, B. Princípios de Marketing. São Paulo: ATLAS, 1983

GALEMBECK, F.; CSORDAS, Y. Cosméticos: a química da beleza. 2015.

KARSAKLIAN, Eliane. Comportamento do consumidor. $2^{2}$. ed., $3^{\underline{a}}$ reimp. São Paulo: Atlas, 2008. P. 13, 26, 34, 99-II, 153-157,212 e 213.

KOTLER, Philip; KELLER, Kevin L. Administração de marketing. I2 ${ }^{\underline{a}}$ edição. São Paulo: Pearson Prentice Hall. 2006. P I77-179; I82-186; 532.

LACERDA, T.S. Teorias Da Ação E O Comportamento Do Consumidor: Alternativas E Contribuições Aos Modelos De Fishbein E Ajzen. In: ANPAD, 31. 2007. Rio de Janeiro. Anais...Rio de Janeiro, 2007.

LIMA, A. M. O conceito de análise de comportamento. Psicologado Artigos. docomportamento>.

Maringá, v. 32, n. I, p. 6i-66, 2010.

MINIARD P.; COHEN J. B. Modeling personal and normative influences on behavior. Journal of consumer Research, P. 169-180, Sept. 1983. 
MINISTÉRIO DE SAÚDE. Decreto-Lei n. ${ }^{\mathrm{o}}$ 128/86. Estabelece as regras que disciplinam o mercado dos produtos cosméticos e de higiene corporal. Brasília, I986. ART $2^{\circ}$, cap. I, dec.-lei 128/86, de 03 de Junho.

PROFESSORES DO DEPARTAMENTO DE MERCADOLOGIA DA FGV-EAESP E CONVIDADOS, 2004. Coordenação Sérgio Roberto Dias. Gestão de marketing. I $^{\mathrm{a}}$ edição. São Paulo: Saraiva, 2004. P. 37-94; 272.

PINHEIRO, Roberto M.; CASTRO, Guilherme C.; SILVA, Helder Hj; NUNES, José Mauro G. Comportamento do consumidor e pesquisa de mercado. $3^{\text {a }}$ edição. Rio de Janeiro: Editora FGV, 2006. P 21; 37.

SANFELICE, Andréia Maria; TRUITI, Maria da Conceição Torrado. Produtos em filme inovação na tecnologia de cosméticos. Revista Acta Scientiarum - Health Sciences, SCHMITT, Bernd. SIMONSON, Alex. A Estética do Marketing. São Paulo: Nobel, 20oz; SURVEYMONKEY. Soluções de Pesquisas Online. Disponível em Acesso em 28 set. 2021.

SOLOMON, M.R. O comportamento do consumidor: comprando, possuindo e sendo. Porto Alegre: BOOKMAN, 2oIr.

RICHERS, Raimer. O Enigmático mas Indispensável Consumidor: teoria e prática. Revista da Administração, Jul/Set I984;

SÁ. De S. Cosméticos brasileiros vivem momento de expansão. Mundo do Marketing.

SCHMITT, Bernd. SIMONSON, Alex. A Estética do Marketing. São Paulo: Nobel, 2002; SURVEYMONKEY. Soluções de Pesquisas Online.

ZUCCO A, SOUSA F. S, ROMEIRO M. COSMÉTICOS NATURAIS: UMA OPÇÃO DE INOVAÇÃO SUSTENTÁVEL NAS EMPRESAS. 2012. 